\title{
APLICACIÓN DE LA PROGRAMACIÓN LÓGICA EN LA IDENTIFICACIÓN DE PECES MARINOS PERUANOS
}

\author{
Ricardo Vilchez Chumacero ${ }^{1}$
}

\begin{abstract}
RESUMEN
El presente trabajo propone una combinación de las redes semánticas y la programación lógica en la identificación de peces marinos peruanos. De la red semántica de peces marinos, se completo las secciones de Lampreas y Quimeras, para su codificación en SWI-Prolog (versión 5.02), creándose una base de conocimientos de especies ícticas marinas peruanas.
\end{abstract}

Palabras Clave: Red semántica, pez, Prolog, base de conocimientos.

\section{ABSTRACT}

The present work proposes a combination of the semantic nets and the logical programming in the identification of Peruvian marine fish. Of the semantic net of marine fish, you complete the sections of Lampreys and Chimeras, for their code in SWI-Prolog (version 5.02), being created a base of knowledge of species fish marine Peruvians.

Key words: Semantic net, fish, Prolog, base of knowledge.

\section{INTRODUCCIÓN}

La inteligencia humana es en un extremo compleja y mucho más amplia que la de las computadoras. Un factor clave que distingue a los seres humanos de otros animales es su capacidad para desarrollar asociaciones y usar metáforas y analogías. Al hacerlo los seres humanos crean reglas nuevas, aplican reglas viejas a situaciones nuevas $y$, a veces, actúan de forma intuitiva o instintiva, sin reglas. Gran parte de lo que se llama sentido común o generalidad en las personas radica en la capacidad para crear metáforas y analogías.

En áreas del saber como clasificar especimenes biológicos, es posible entender, codificar y colocar en una computadora las reglas prácticas que usan expertos del mundo real. Los sistemas de información que resuelven problemas capturando conocimiento de un dominio muy específico y limitado del saber humano, se denominan sistemas expertos. Estos ayudan a tomar decisiones, pues hacen preguntas pertinentes y explican las razones para efectuar ciertas acciones.

El conocimiento humano se debe modelar o representar en una forma que una computadora logre procesar. El modelo de conocimiento humano que un sistema experto utiliza se denomina base de conocimientos. Las reglas y los marcos de conocimiento son las dos formas de representar los conocimientos y la experiencia de los seres humanos. Sería posible representar los conocimientos contenidos en la Clave para identificar peces marinos del Perú. La repuesta la encontraremos al final de este articulo.

\section{MATERIAL Y MÉTODOS}

Las redes semánticas son estructuras que codifican el conocimiento taxonómico sobre objetos y propiedades de estos. Se trata de un lenguaje gráfico, fácilmente traducible a forma simbólica, muy útil para representar conocimiento taxonómico, es decir, aquel que permite agrupar los elementos del universo en una jerarquía de clases y subclases entre las cuales existe una relación de herencia. Este lenguaje tiene la ventaja de permitir una representación estructurada del conocimiento factual y de ciertos tipos de conocimiento normativo, y de expresar de manera muy fácil las excepciones en los valores de propiedades heredades.

La capacidad de construir una red semántica es señal de una comprensión bien desarrollada del tema. Una red semántica es un grafo dirigido en el cual los nodos corresponden a:

Constantes de relación tales como clases y propiedades.

Instancias: Objetos de alguna clase. Un arco en una red semántica puede tener diversas etiquetas:

Instancia de o ID, definidas entre objetos y clases.

Subclase de o SD, definidas entres subclases y clases.

Otras etiquetas que representan atributos de clases que dependen de la información que uno quiera representar. Por Ejemplo, podemos considerar las etiquetas "tiene" 0 " $\mathrm{t}$ " $\mathrm{y}$ "come" o "c".

El motivo de realizar la implementación en Prolog de la red semántica es porque de todos los lenguajes de programación disponibles hoy en día, es uno de los más adecuados para las aplicaciones en la identificación de peces. Para modelar esta red semántica en Prolog, se codifica el grafo que la representa. Una Red Semántica puede ser codificada con un pequeño programa en Prolog en el que los nodos, y los arcos (junto con sus etiquetas) son modelados como conjuntos de cláusulas unitarias.

Se utilizó una computadora personal Pentium III, $700 \mathrm{MHZ}$, 128MB de Memoria RAM. El sistema operativo utilizado es Windows 98. La implementación fue desarrollada en SWI-Prolog (Version 5.0.2) de la Universidad de Ámsterdam http://www. swi-prolog.org

\footnotetext{
${ }^{1}$ Universidad Faustino Sánchez Carrión. E-mail: rivich@hotmail.com
} 


\section{RESULTADOS}

Al construir el modelo de bloques, tomado como base de hechos los contenidos en la Clave para identificar peces marinos del Perú, puede ser visto a través de tres niveles. En el nivel más alto los peces marinos, en el nivel medio las 5 secciones de peces y en el nivel mas bajo 673 especies. La distribución por secciones es:

A (Lampreas) 2 especies,

B (Tiburones) 57 especies,

C (Rayas) 40 especies,

D (Quimeras) 5 especies y

E (Peces Óseos) 569 especies.

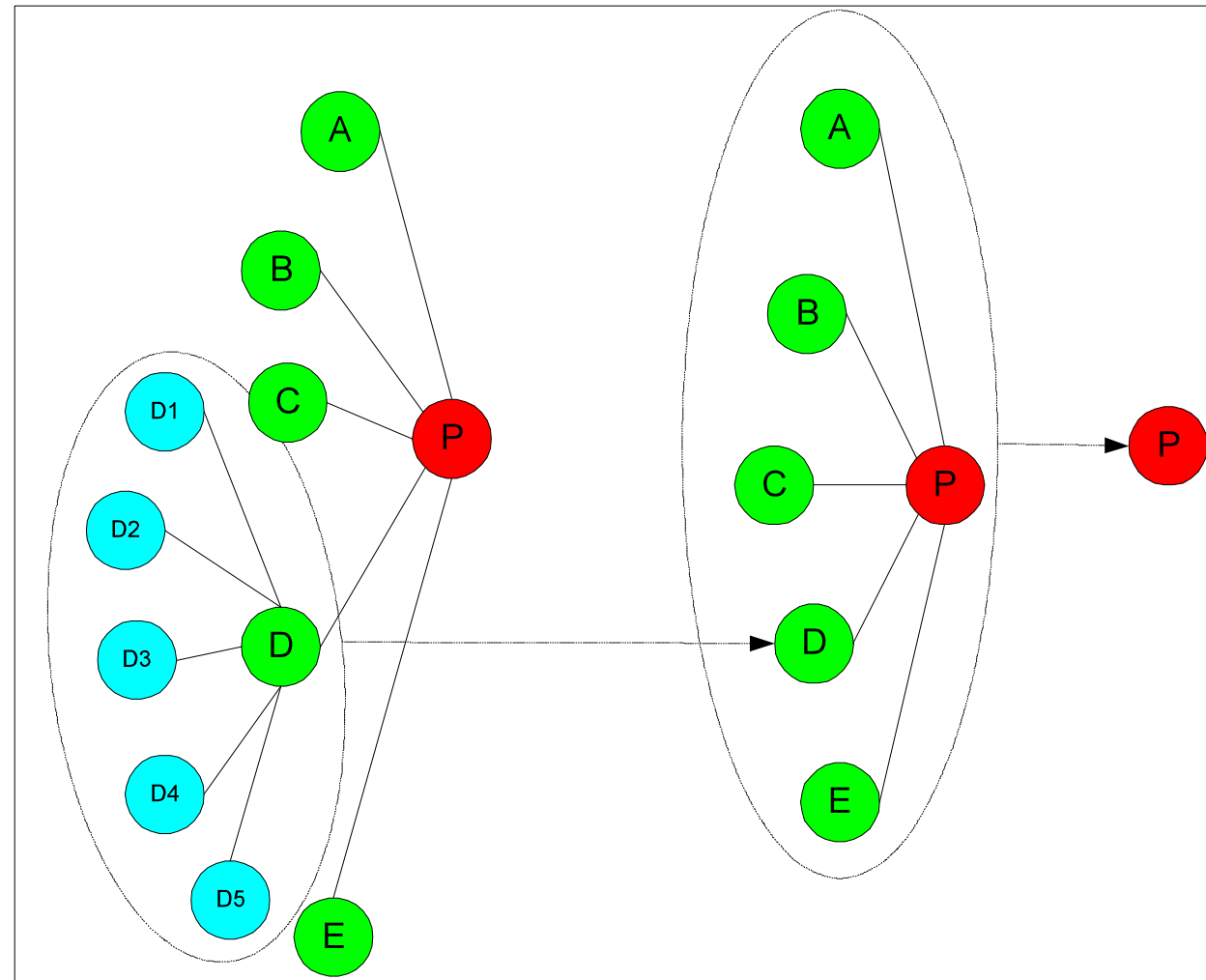

Figura 1. Modelo de bloques de Peces Marinos Peruanos

En la Figura 1 muestra que para crear programas lógicos, es necesario construir una serie de niveles de bloques, a fin de ir hacia el nivel más bajo es necesario cumplir con requisitos en distintos niveles de una mismo bloque. En la construcción de la red semántica de peces marinos, se obtiene un grafo con tres niveles. Los nodos elipsoidales representan la raíz (peces), en le primer nivel las clases (secciones: $A, B, C, D$ y E) y el segundo nivel representado por las instancias de clases (especies A1, A2, D1, D2, D3, D4 Y D5). Los nodos rectangulares representan características morfológicas de especies de peces marinos peruanos. En la figura 2 se muestra la red semántica de peces marinos peruanos. Es importante señalar que no se muestra en detalle las secciones $B, C$ y $E$.
El objetivo es llegar a las especies pasando por cada uno de los niveles de características morfológicas. Una de las ramas mas corta es Especie A1, que corresponde la Sección A (Lampreas) y su nombre es Myxine circifrons. La Figura 2 muestra que para llegar a identificar la Especie A1, tiene que pasar dos niveles que corresponden a los caracteres morfológicos PA1 y A1a. La Sección $D$ de Quimeras tiene 5 especies (D1, D2, D3, D4 y D5). Las características morfológicas son tomados de la Clave para identificar peces marinos del Perú, basada en el sistema dicótomo

A1a Los tres primeros dientes linguales de la serie anterior fusionados en su base; cuerpo negro, cabeza más clara cerca de su boca.

A1b Los dos primeros dientes linguales de la serié anterior fusionados en su base.

A1 Myxine circifrons Garman. "Lamprea de mar". "Lamprea babosa". "Whiteface hagfish".

A2 Myxine sp. "Babosa". "Hagfish"

\section{Base de hechos}

Para lograr su codificación en SWIProlog ingresaremos los siguientes hechos:

Primero declararemos el vínculo de las características morfológicas P1a y P1b con la raíz PEZ

subclase_de(p1a,pez).

subclase_de(p1b,pez).

De la misma manera vamos descendiendo en la red semántica y conectamos P1a y P1b con sus correspondientes nodos:, hasta llegar a las secciones $A, B, C, D$ y $E$.

subclase_de(seccionA,p1a).

subclase_de(p2a,p1b).

subclase_de(p2b,p1b)

subclase_de(p3a,p2a)

subclase_de(p3b,p2a).

subclase_de(p4a,p2b).

subclase_de(p4b,p2b)

subclase_de(seccionB,p3a).

subclase_de(seccionC,p3b).

subclase_de(seccionD,p4a).

subclase_de(seccionE,p4b).

con dos alternativas de caracteres "a" y "b". Cada sección se enumera independiente en forma correlativa al cual se agregado la codificación de LETRA-NUMERO-ALTERNATIVA, y las especies ícticas marinas peruanas codificadas con sección y numero de identificación (LETRA-NUMERO)

P1a Boca sin mandíbulas, con un disco succionador a manera de ventosa, rodeado de barbos y con pequeñas placas córneas a manera de dientes; sin aletas pectorales y pélvicas; ojos no visibles externamente.

P1b Boca con mandíbulas bien desarrolladas y armadas generalmente con dientes
Ampliamos las características morfológicas hasta su conexión con las respectivas secciones

\section{SECCION A}

subclase_de(a1a, seccionA). subclase_de(a1b, seccionA).

\section{SECCION D}

subclase_de(d1a, seccionD).

subclase_de(d1b, seccionD).

subclase_de(d2a,d1b)

subclase_de(d2b,d1b).

subclase de(d3a,d2a).

subclase de (d3b,d2a).

subclase_de(d4a,d2b)

subclase_de(d4b,d2b).

Ahora declararemos las instancias de clase u objetos a los cuales corres- 


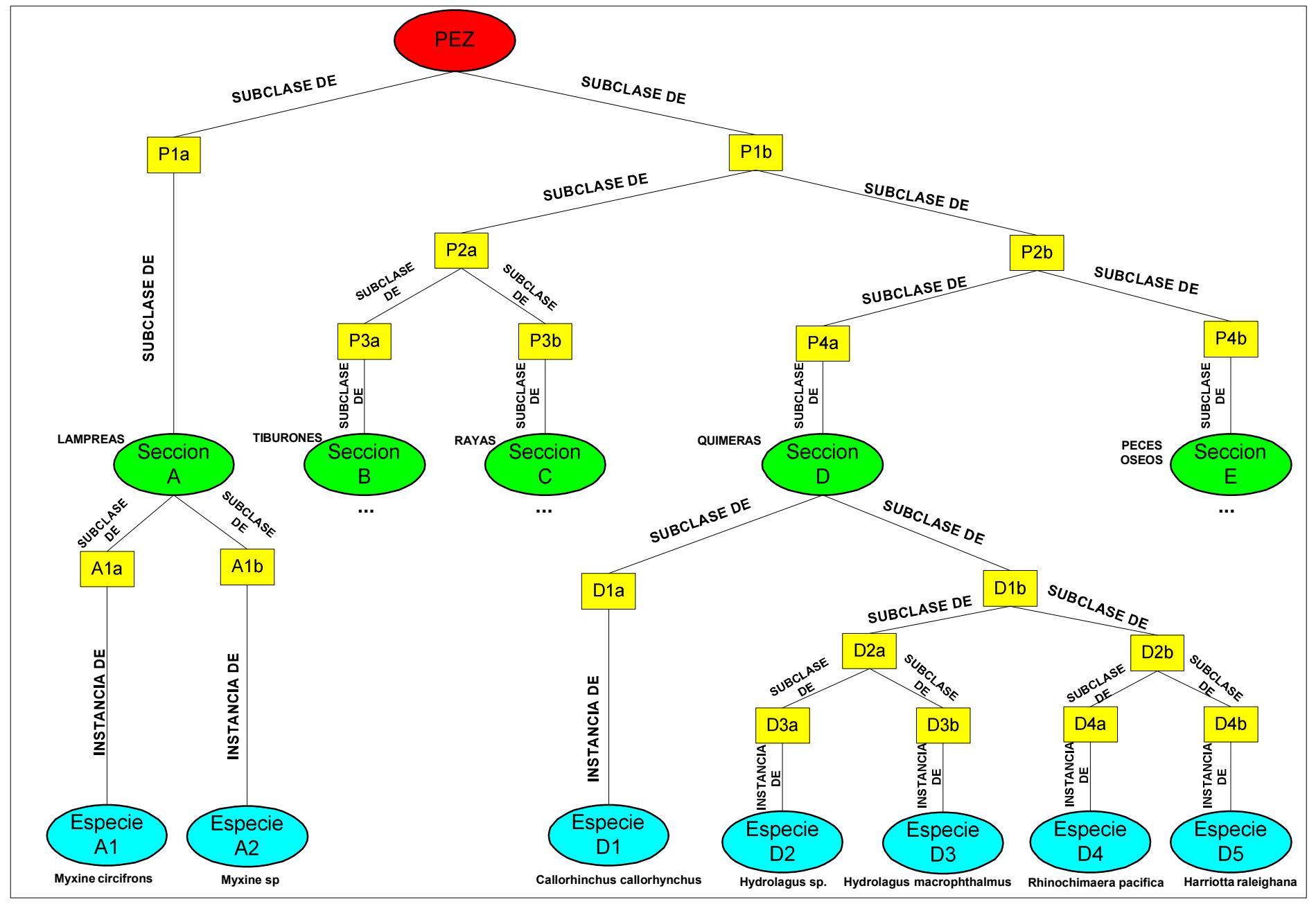

FIGURA 2. Red Semántica de Peces Marinos peruanos.

ponden las especies ícticas marinas peruanas, conectados con su característica morfológica.

\section{SECCIÓN A:}

instancia_de(a1,a1a).

instancia_de(a2,a1b).

SECCIÓN D:

instancia_de(d1,d1a).

instancia_de(d2,d3a).

instancia_de(d3,d3b).

instancia de $(\mathrm{d} 4, \mathrm{~d} 4 \mathrm{a})$.

instancia_de(d5,d4b).

\section{Reglas}

Una vez terminado el ingreso de las sentencias estableceremos las reglas que permiten al programa dar respuesta a nuestras consultas.

Regla 1: Sirve para determinar todas las especies de peces y para mostrar el camino desde la especie hasta la raíz.

es(Clase,Obj):-instancia_de(Obj,Clase).

es(Clase,Obj):-instancia_de(Obj,Clasep)

$\%$ ?- es(pez,X) descendientes(Clasep,Clase).

$\%$ ?- es $(X, d 5)$.

Regla 2: Determinara todos los descendientes desde cualquier nodo hasta las especies.

Descendientes $(C 1, C 2)$ :-subclase_de $(C 1, C 2)$.

descendientes $(C 1, C 2)$ :-subclase_de $(C 1, C 3)$, descendientes $(C 3, C 2)$;es $(C 2, C 1)$.

$\%$ ?- descendientes (X,p1a).

Regla 3: Muestra todos los ancestros de los cuales desciende un nodo.

ancestros $(C 2, C 1)$ :- subclase_de $(C 1, C 2)$

ancestros(C2,C1):-subclase_de(C1,C3),

$\%$ ?- $\operatorname{ancestros}(\mathrm{X}, \mathrm{d} 3 \mathrm{a})$. ancestros(C2,C3);es(C2,C1)

Regla 4: Visualiza el nodo hermano del nodo que ingresemos

hermano(A1, A2):-subclase_de $(A 1, Z)$, subclase_de $(\mathrm{A} 2, \mathrm{Z}), \mathrm{A} 1 \mathrm{l}=\mathrm{A} 2$.

\%?- hermano(X,p1b).

Regla 5: Sive para mostrar las especies por secciones especies(C2,C1):-descendientes(C2,C1).

especiesm(C2,C1):-write('Son los siguientes:'), $\mathrm{nl}$, es $(\mathrm{C} 1, \mathrm{C} 2)$.

$\%$ ?- especiesm(X,seccionD).

Regla 6: Permite visualizar los nodos hijos desde un nodo dado sin mostrar las especies.

especiesb(C2,C1):-descendientes(C2,C1), not(es(C1,C2)). $\%$ ?- especiesb(X, seccionD).

Regla 7: Visualiza el recorrido entre dos nodos de una misma rama.

estan_entre $(C 1, C 3, C 2)$ :-descendientes $(C 3, C 1)$, especies $(\mathrm{C} 2, \mathrm{C} 3)$; descendientes $(\mathrm{C} 1, \mathrm{C} 3)$, especies $(\mathrm{C} 3, \mathrm{C} 2)$.

$\%$ ?- estan_entre(pez, $\mathrm{X}, \mathrm{a} 1)$.

Regla 8: Permite cambiar los nombres de los nodos. intercambiar(X, Y):-X=pez,Y='PEZ MARINO PERUANO'. 
intercambiar $(X, Y):-X=p 1 a, Y='(p 1 a)$ Boca sin mandíbulas, con un disco succionador a manera de ventosa, rodeado de barbos y con pequeñas placas córneas a manera de dientes; sin aletas pectorales y pélvicas; ojos no visibles externamente.'.

intercambiar $(X, Y):-X=$ seccion $A, Y=$ lampreas.

intercambiar $(X, Y):-X=a 1 a, Y={ }^{\prime}(a 1 a)$ Los tres primeros dientes linguales de la serie anterior fusionados en su base; cuerpo negro, cabeza más clara cerca de su boca.'.

intercambiar(X, $\mathrm{Y}):-\mathrm{X}=\mathrm{a} 1 \mathrm{~b}, \mathrm{Y}=\mathrm{\prime}^{\prime}(\mathrm{a} 1 \mathrm{~b})$ Los dos primeros dientes linguales de la serie anterior fusionados en su base.'.

intercambiar $(X, Y):-X=a 1, Y=$ '(a1) Myxine circifrons'.

intercambiar(X, Y):-X=a2, $Y={ }^{\prime}(a 2)$ Myxine sp'.

Regla 9: Cambia los nombres de las instancias de la Sección.

es_1(C1,C2):-instancia_de(C2,C1).

es_1(C1,N):-instancia_de $(C 2, C 3)$, subc_1(C3,C1), inter-

cambiar( $\mathrm{C} 2, \mathrm{~N})$.

subc_1(X,Y):-subclase_de $(X, Y)$.

subc_1(X,Y):-subclase_de(X,Z), subc_1(Z,Y).

$\%$ ?-ề_1(seccionA, X).

Regla 10: Permite cambiar la rama izquierda de la red semántica

es_2(C1,C2):-instancia_de(C2,N), intercambiar(N,C1).

es_2(C1,C2):-instancia_de(C2,C3), subc_2(C3,C1).

subc_2(X,Y):-subclase_de $(X, N)$, intercambiar

$(\mathrm{N}, \mathrm{Y}), \mathrm{XI}=$ 'p1b'.

subc_2(X,Y):-subclase_de $(X, Z)$,subc_2(Z,Y);es_2(Y,X).

$\%$ ?- es_2(X,a1).

\section{DISCUSIÓN}

Si es posible crear una Base de Hechos contenidos en la Clave para identificar peces marinos del Perú. Se diseño la red semántica de peces, el motivo de realizar la implementación de Prolog es su facilidad para realizar representaciones del conocimiento de especies ícticas. Además se crean diez reglas que junto con la base de hechos forman una Base de Conocimientos de los peces, que constituye la base de trabajos futuros de taxonomía o la creación de un Sistema Experto.

\section{CONCLUSIONES}

- Las redes semánticas y la programación lógica son aplicables para la identificación de peces.

- Abre las posibilidades de almacenar información en forma activa y capacitar a las personas relacionadas con el sector pesquero.

- Es factible realizar representaciones del conocimiento con facilidad construyendo redes semánticas para el manejo de gran cantidad de información en forma sencilla y rápida.

\section{REFERENCIAS BIBLIOGRAFICAS}

1. Chirichigno N, Cornejo M. Catálogo comentado de los peces marinos del Perú. Ed. Imarpe: Callao; 2001.

2. Chirichigno N, Vélez J. Clave para Identificar los Peces Marinos del Perú. Informe $\mathrm{N}^{\circ}$ 44. 2nd rev ed. Imarpe: Callao; 1998.

3. Giarratano J, Riley G. Sistemas Expertos: Principios y programación. 3ra. Ed. International Thomson: México D.F; 2001.

4. Hofstadter D. Gödel, Escher, Bach un Eterno y Grácil Bucle. Ed. Turquets: Barcelona; 1992.

5. Laudon K, Laudon J. Sistemas de Información Gerencial. Organización y Tecnología de la Empresa Conectada en Red. Ed. Pearson: México D.F.; 2002.

6. Munera, Luis. Principios de Inteligencia Artificial y Sistemas Expertos. Ed. Universidad ICESI: Madrid; 2001.

7. Nilsson, Nils. Inteligencia Artificial. Una nueva síntesis. Ed. McGraw-Hill: Madrid; 2001.

8. Russell S, Norvig, P. Inteligencia Artificial: Un enfoque moderno. Ed. Prentice Hall: México D.F; 1996. 